\title{
Interaction potential between vortex lines for uniaxial superconductors in the London approximation
}

\author{
Edson Sardella \\ Departamento de Física, Universidade Estadual Paulista, \\ Caixa Postal 473, 17033-360, Bauru-SP, Brazil
}

(Received 30 June 1995; revised manuscript received 19 January 1996)

\begin{abstract}
Two distinct expressions of the interaction potential between arbitrarily oriented curved vortex lines with respect to the crystal $c$ axis are derived within the London approximation. One of these expressions is used to compute the eigenvalues of the elasticity matrix. We examine the elastic properties of the vortex chain lattice, recently proposed, concerning shearing deformation. [S0163-1829(96)04821-7]
\end{abstract}

\section{INTRODUCTION}

The equilibrium configuration of the vortex lattice for high- $T_{c}$ superconductors has been of great interest since these materials were discovered. The high- $T_{c}$ superconductors are strongly anisotropic compounds. The anisotropy introduces many interesting novelties in the properties of the vortex lattice, affecting its geometry and introducing strange and yet unexpected features as the angle $\theta$ between the induction $\mathbf{B}$ (the average vortex direction) and the crystal $c$ axis is changed. A great deal of experimental and theoretical works ${ }^{1-4}$ have been dedicated to investigate the shape of the vortex lattice for arbitrarily oriented $\mathbf{B}$.

For isotropic superconductors, the interaction between the vortices is repulsive. The anisotropy modifies the interaction between the vortices as one varies the angle $\theta$ and the anisotropy and may even become attractive. ${ }^{5}$ This may affect the symmetry of the equilibrium configuration of the vortex lattice.

Campbell, Doria, and Kogan ${ }^{4}$ found that in the regime of high induction (well above the lower critical field $H_{c 1}$ ), the equilibrium configuration of the vortex lattice of an anisotropic superconductor is a deformed triangular lattice in which the nearest-neighbor distances between the vortices vary in a simple manner with the anisotropy, the angle $\theta$, and induction $B$.

The study of the elastic properties of the vortex lattice demands knowledge of the correct geometry of the vortex lattice. The use of geometries which do not correspond to the equilibrium configuration of the vortex lattice may lead to negative values of the elastic moduli. Sudb $\varnothing$ and Brandt, ${ }^{6}$ for instance, have found that the shear modulus becomes negative to sufficient high anisotropy and low induction by using the deformed triangular lattice mentioned above. This signals a structural instability of the vortex lattice.

Daemen, Campbell, and Kogan ${ }^{3}$ proposed a type of vortex lattice for the low-induction regime. The vortex lattice is still a deformed triangular lattice. However, nearest-neighbor distances vary in a nontrivial form with the angle $\theta$, the anisotropy, and the induction $B$. For low induction, the external magnetic field penetrates the sample in the form of chains of vortices in which the distance between vortices intrachain does not vary with $B$ and the distance between adjacent chains goes as $1 / B$. Quite different behavior at high induction results.

The study of the elastic properties of the vortex lattice requires not only knowledge of its equilibrium configuration but also the expression of the three-dimensional (3D) interaction potential between the vortex lines. In Ref. 7 a $2 \mathrm{D}$ straight-vortex line interaction has been proposed in reciprocal space and its generalization to the three-dimensional case in Ref. 8 for anisotropic uniaxial superconductors and the induction $\mathbf{B}$ tilted away from the crystal $c$ axis by an arbitrary angle $\theta$. In real space, an expression for 3D interactions has been derived by Sudb $\varnothing$ and Brandt ${ }^{9}$ for the simple geometry $\theta=0$. Here we generalize their result for arbitrary $\theta$. We also present an alternative expression for the 3D interaction potential in reciprocal space by using the fact that the vorticity is divergence free.

As an application of this alternative expression for the interaction potential we investigate the elastic properties of the vortex chain lattice with respect to shearing deformation. We also analyze the stability of this lattice against uniform shear deformation.

\section{INTERACTION POTENTIAL}

In this section we discuss the interaction potential of any arrangement of curved London vortex lines for an anisotropic superconductor with uniaxial symmetry. London theory is valid in the limit in which the fields penetrate over a distance which is much larger than the size of the vortex core. Within this approximation, the depression of the superconducting order parameter near the vortex core may be neglected and the second Ginzburg-Landau equation can be linearized and solved exactly. Thus, the free energy of an ensemble of curved London vortex lines can be expressed as

$$
\begin{aligned}
F & =\frac{\Phi_{0}^{2}}{8 \pi} \sum_{i, j} \int d r_{i}^{\alpha} \int d r_{j}^{\beta} V_{\alpha \beta}\left(\mathbf{r}_{i}-\mathbf{r}_{j}\right) \\
& =\frac{\Phi_{0}^{2}}{8 \pi} \int d^{3} r \int d^{3} r^{\prime} \nu_{\alpha}(\mathbf{r}) V_{\alpha \beta}\left(\mathbf{r}-\mathbf{r}^{\prime}\right) \nu_{\beta}\left(\mathbf{r}^{\prime}\right) \\
& =\frac{\Phi_{0}^{2}}{8 \pi} \int \frac{d^{3} k}{(2 \pi)^{3}} \widetilde{\nu}_{\alpha}(-\mathbf{k}) \widetilde{V}_{\alpha \beta}(\mathbf{k}) \widetilde{\nu}_{\beta}(\mathbf{k}),
\end{aligned}
$$

where we have used the usual summation over the indices repeated twice and $\Phi_{0}$ is the quantum flux. The sum in (1) 
runs over all vortex elements $d \mathbf{r}_{i}, d \mathbf{r}_{j}$ including $i=j$, although in this case a cutoff scheme is required to account for the finite vortex core [see (5)-(9) below]. The Fourier transform of the interaction (tensor) potential $V_{\alpha \beta}(\mathbf{r})$, for superconductors with uniaxial symmetry, is given by

$$
\widetilde{V}_{\alpha \beta}(\mathbf{k})=\frac{1}{\left(1+\Lambda_{1} k^{2}\right)}\left[\delta_{\alpha \beta}-\frac{\Lambda_{2} q_{\alpha} q_{\beta}}{1+\Lambda_{1} k^{2}+\Lambda_{2} q^{2}}\right],
$$

where $\mathbf{q}=\mathbf{k} \times \mathbf{c}, \Lambda_{1}=\Lambda_{a b}^{2}, \Lambda_{2}=\lambda_{c}^{2}-\Lambda_{a b}^{2}$, and $\lambda_{a b}, \lambda_{c}$ are the in- and out-of-plane penetration lengths, respectively. ${ }^{7,8}$ On going from the first to the second line in (1) we have used the following definition for the vorticity:

$$
\nu(\mathbf{r})=\sum_{i} \delta^{(2)}\left[\mathbf{r}-\mathbf{r}_{i}(z)\right] \frac{d \mathbf{r}_{i}(z)}{d z},
$$

where $\mathbf{r}_{i}(z)$ is the position of the $i$ th vortex line at a certain height $z$ above the $x y$ plane. Notice that the system of coordinates used in the equations above is the vortex frame in which the average vortex direction (the induction) $\mathbf{B} \| z$ lies in the $x z$ plane and is tilted away from the $c$ axis by an angle $\theta$.

The interaction potential of (1),

$$
V_{\alpha \beta}(\mathbf{r})=\int \frac{d^{3} k}{(2 \pi)^{3}} \widetilde{V}_{\alpha \beta}(\mathbf{k}) e^{i \mathbf{k} \cdot \mathbf{r}},
$$

has been evaluated in Ref. 9 for the simple geometry $z \| c$. In the Appendix we present the derivation of this potential which is an extension of the result of Ref. 9 for the case of an inclined average vortex direction $(\theta \neq 0)$. The result is

$$
V_{\alpha \beta}(\mathbf{r})=V_{1}^{\alpha \beta}(\mathbf{r})+V_{2}^{\alpha \beta}(\mathbf{r}),
$$

where

$$
\begin{aligned}
& V_{1}^{\alpha \beta}(\mathbf{r})=\frac{\delta_{\alpha \beta}}{4 \pi \lambda_{a b}^{2}} \frac{e^{-r / \lambda_{a b}}}{r}, \\
& V_{2}^{\alpha \beta}(\mathbf{r})= \frac{1}{4 \pi \lambda_{a b}(\mathbf{r} \times \hat{\mathbf{c}})^{2}}\left[G_{1}(\mathbf{r})\left(\delta_{\alpha \beta}-c_{\alpha} c_{\beta}\right)\right. \\
&\left.-G_{2}(\mathbf{r}) \frac{(\mathbf{r} \times \hat{\mathbf{c}})_{\alpha}(\mathbf{r} \times \hat{\mathbf{c}})_{\beta}}{(\mathbf{r} \times \hat{\mathbf{c}})^{2}}\right], \\
& G_{1}(\mathbf{r})= e^{-r / \lambda_{a b}-e^{-\sqrt{(\mathbf{r} \times \hat{\mathbf{c}})^{2}+\epsilon^{2}(\mathbf{r} \cdot \hat{\mathbf{c}})^{2}} / \lambda_{c}},} \\
& G_{2}(\mathbf{r})=\left(2+\frac{(\mathbf{r} \times \hat{\mathbf{c}})^{2}}{\lambda_{a b} r}\right) e^{-r / \lambda_{a b}} \\
&-\left(2+\frac{(\mathbf{r} \times \hat{\mathbf{c}})^{2}}{\lambda_{c} \sqrt{(\mathbf{r} \times \hat{\mathbf{c}})^{2}+\epsilon^{2}(\mathbf{r} \cdot \hat{\mathbf{c}})^{2}}}\right) \\
& \times e^{-\sqrt{(\mathbf{r} \times \hat{\mathbf{c}})^{2}+\epsilon^{2}(\mathbf{r} \cdot \hat{\mathbf{c}})^{2} / \lambda_{c}},}
\end{aligned}
$$

and $\epsilon=\lambda_{c} / \lambda_{a b}$ is the anisotropy.

Notice that if we take the particular geometry $z \| c$ $(\mathbf{B} \| c)$ one has $(\mathbf{r} \times \hat{\mathbf{c}})^{2}=x^{2}+y^{2}=\rho^{2}, \quad(\mathbf{r} \cdot \hat{\mathbf{c}})^{2}=z^{2} \quad$ and $(\mathbf{r} \times \hat{\mathbf{c}})_{\alpha}(\mathbf{r} \times \hat{\mathbf{c}})_{\beta}=\boldsymbol{\epsilon}_{\alpha j l} \boldsymbol{\epsilon}_{\beta m n} c_{l} c_{n} x_{j} x_{m}=\epsilon_{\alpha j z} \epsilon_{\beta m z} x_{j} x_{m}=\delta_{\alpha \beta} \rho^{2}$ $-x_{\alpha} x_{\beta}$, now with $\alpha, \beta=x, y$. Equations (5)-(10) of Ref. 9 are then recovered. Note also that both the isotropic (6) and anisotropic (7) parts of (5) are singular at $\mathbf{r}=0$. To overcome this difficulty one has to use some model for the vortex core.

The expressions for the interaction potential of London vortices (5) -(9) may be very useful in a wide range of physical applications. Sudb $\varnothing$ and Brandt ${ }^{10}$ have evaluated the energy barrier for mutual cutting of twisted vortex lines and also for a pair of rigid straight-vortex lines. They assume that the symmetry axis between the vortices is parallel to the $c$ axis $[\theta=0$ in $(5)-(9)]$. They claim that the cutting barrier is very small and under certain circumstances even negative, indicating that the entangled configuration is more stable. The same problem for the situation in which the symmetry axis is tilted away from the $c$ axis will be left to a further contribution. ${ }^{11}$ In addition, (5)-(9) could be very useful to determine field and current distribution by using the expression

$$
H_{\alpha}(\mathbf{r})=\Phi_{0} \sum_{i} \int d r_{i}^{\beta}\left(z^{\prime}\right) V_{\alpha \beta}\left[\mathbf{r}-\mathbf{r}_{i}\left(z^{\prime}\right)\right]
$$

This representation of the interaction potential of (2) is not uniquely defined since the vorticity is divergence free, that is, $\mathbf{k} \cdot \widetilde{\nu}(\mathbf{k})=0$, which means that there are no sources or sinkholes of vortex lines. ${ }^{12}$ By introducing this expression into (1) and using (2), we obtain, after length algebra,

$$
\begin{gathered}
\widetilde{V}_{x x}(\mathbf{k})=\frac{1+\left(\Lambda_{1}+\Lambda_{2} \sin ^{2} \theta\right) k^{2}}{\left(1+\Lambda_{1} k^{2}\right)\left(1+\Lambda_{1} k^{2}+\Lambda_{2} q^{2}\right)}, \\
\widetilde{V}_{y y}(\mathbf{k})=\frac{1}{\left(1+\Lambda_{1} k^{2}+\Lambda_{2} q^{2}\right)}, \\
\widetilde{V}_{z z}(\mathbf{k})=\frac{1+\left(\Lambda_{1}+\Lambda_{2} \cos ^{2} \theta\right) k^{2}}{\left(1+\Lambda_{1} k^{2}\right)\left(1+\Lambda_{1} k^{2}+\Lambda_{2} q^{2}\right)}, \\
\widetilde{V}_{x z}(\mathbf{k})=\widetilde{V}_{z x}(\mathbf{k})=-\frac{\cos \theta \sin \theta \Lambda_{2} k^{2}}{\left(1+\Lambda_{1} k^{2}\right)\left(1+\Lambda_{1} k^{2}+\Lambda_{2} q^{2}\right)},
\end{gathered}
$$

and zero otherwise. These expressions can also be written as

$$
\begin{gathered}
\widetilde{V}_{x x}(\mathbf{k})=\left[1+\frac{\Lambda_{2}}{\Lambda_{1}} \sin ^{2} \theta\right] \widetilde{U}(\mathbf{k})-\frac{\sin ^{2} \theta}{\Lambda_{1}} \widetilde{I}(\mathbf{k}), \\
\widetilde{V}_{y y}(\mathbf{k})=\widetilde{U}(\mathbf{k}), \\
\widetilde{V}_{z z}(\mathbf{k})=\left[1+\frac{\Lambda_{2}}{\Lambda_{1}} \cos ^{2} \theta\right] \widetilde{U}(\mathbf{k})-\frac{\cos ^{2} \theta}{\Lambda_{1}} \widetilde{I}(\mathbf{k}), \\
\widetilde{V}_{x z}(\mathbf{k})=-\frac{\cos \theta \sin \theta}{\Lambda_{1}}\left[\Lambda_{2} \widetilde{U}(\mathbf{k})-\widetilde{I}(\mathbf{k})\right],
\end{gathered}
$$

where

$$
\begin{gathered}
\widetilde{U}(\mathbf{k})=\frac{1}{1+\Lambda_{1} k^{2}+\Lambda_{2} q^{2}}, \\
\widetilde{I}(\mathbf{k})=\frac{\Lambda_{2}}{\left(1+\Lambda_{1} k^{2}\right)\left(1+\Lambda_{1} k^{2}+\Lambda_{2} q^{2}\right)} .
\end{gathered}
$$


Notice that at large wave vectors, $\widetilde{U}(\mathbf{k})$ falls off as $1 / k^{2}$ whereas $\widetilde{I}(\mathbf{k})$ goes as $1 / k^{4}$. Thus, $U(\mathbf{r})$ is not well defined at $\mathbf{r}=0$ and a cutoff procedure must be used for the first part of (15)-(18). On the other hand, by the same reason, $I(\mathbf{r}=0)$ is finite and the second part of these equations has nothing to do with a cutoff procedure to make London theory finite. One has

$$
\begin{gathered}
U(\mathbf{r})=\frac{1}{4 \pi \lambda_{a b} \lambda_{c}} \frac{e^{\sqrt{(\mathbf{r} \times \hat{\mathbf{c}})^{2}+\epsilon^{2}(\mathbf{r} \cdot \hat{\mathbf{c}})^{2}} \lambda_{c}}}{\sqrt{(\mathbf{r} \times \hat{\mathbf{c}})^{2}+\epsilon^{2}(\mathbf{r} \cdot \hat{\mathbf{c}})^{2}}}, \\
I(\mathbf{r})=\int \frac{d^{3} k}{(2 \pi)^{3}} \widetilde{I}(\mathbf{k}) e^{i \mathbf{k} \cdot \mathbf{r}} .
\end{gathered}
$$

To remove this short-length scale divergence in $U(\mathbf{r})$ we multiply $\widetilde{U}(\mathbf{k})$ of (19) by an elliptical Gaussian cutoff $e^{-2 G\left(\mathbf{k}_{\perp}\right)}$ where $G\left(\mathbf{k}_{\perp}\right)=\xi_{a b}^{2}\left(\mathbf{k}_{\perp} \times \hat{\mathbf{c}}\right)^{2}+\xi_{c}^{2}\left(\mathbf{k}_{\perp} \cdot \mathbf{c}\right)^{2}$, where $\xi_{a b}$ and $\xi_{c}$ are the in- and out-of-plane coherence lengths, respectively. The identity $\xi_{a b} \lambda_{a b}=\xi_{c} \lambda_{c}$ holds, and $\kappa=\lambda_{a b} / \xi_{a b}$ is the Ginzburg-Landau parameter. (The main steps on how to build this cutoff scheme are sketched in Ref. 13).

Notice also that on going from the first to the second representation we gain in reducing from nine to only five elements of the interaction (tensor) potential and obtaining expressions which are less cutoff dependent. However, in this alternative representation, it seems hardly possible that $I(\mathbf{r})$ can be expressed in terms of elementary functions, though its gradient can be calculated exactly as shown in the Appendix. That is to say that a real space expression for this second representation of $V_{\alpha \beta}(\mathbf{r})$ cannot be written in terms of elementary functions like in (5)-(9). In the next section we evaluate the elasticity matrix by using this alternative representation of the interaction potential.

\section{APPLICATION}

Suppose that initially the vortex lines are parametrized by $z$, the coordinate along the axis orthogonal to the $x y$ plane, $\mathbf{R}_{i}(z)=\left(X_{i}, Y_{i}, z\right)$. Let us denote by $\mathbf{u}_{i}(z)$ the displacement of the $i$ th vortex line from its equilibrium position. The new position of the $i$ th vortex line is then given by $\mathbf{r}_{i}(z)=\mathbf{R}_{i}(z)+\mathbf{u}_{i}(z)$. By introducing this expression into (1) and expanding the free energy in powers of small displacements, and using nonlocal elasticity theory, ${ }^{14}$ after length algebra we find that, up to second order,

$$
\Delta F=\frac{1}{2} \int \frac{d k^{3}}{(2 \pi)^{3}} \tilde{u}_{\alpha}(-\mathbf{k}) \Phi_{\alpha \beta}(\mathbf{k}) \tilde{u}_{\beta}(\mathbf{k}),
$$

where

$$
\begin{gathered}
\Phi_{\alpha \beta}(\mathbf{k})=\frac{B^{2}}{4 \pi} \sum_{\mathbf{Q}}\left[f_{\alpha \beta}(\mathbf{k}+\mathbf{Q})-f_{\alpha \beta}(\mathbf{Q})\right], \\
f_{\alpha \beta}(\mathbf{k})=k_{z}^{2} \widetilde{V}_{\alpha \beta}(\mathbf{k})+k_{\alpha} k_{\beta} \widetilde{V}_{z z}(\mathbf{k})-2 k_{z} k_{\alpha} \widetilde{V}_{z \beta}(\mathbf{k}),
\end{gathered}
$$

where $\mathbf{Q}$ are the reciprocal lattice vectors and $\widetilde{\mathbf{u}}(\mathbf{k})$ is the Fourier transform of $\mathbf{u}_{i}(z)$. Notice that for $\mathbf{k}=0, \Delta F=0$, since a uniform displacement costs no energy to the system. Although (23) is valid for any arrangement of vortex lines, we use the lattice predicted by Daemen, Campbell, and Kogan. ${ }^{3}$ For completeness, we outline the main steps of their method of deducing the equilibrium configuration of the vortex lattice.

Call $L_{1}$ and $L_{2}$ the two unit cell sides and $\psi$ the angle between them. The side $L_{1}$ is along the $x$ axis and $L_{2}$ is in the $x y$ plane orthogonal to the induction $\mathbf{B}$ and makes an angle $\psi$ with respect to the $x$ axis. Then, the lattice vectors are given by

$$
\mathbf{R}_{m n}=\left(m L_{1}+n L_{2} \cos \psi\right) \mathbf{x}+n L_{2} \sin \psi \mathbf{y},
$$

where $m, n$ are integer numbers. The corresponding reciprocal lattice vectors are given by

$$
\mathbf{Q}_{m n}=n \frac{2 \pi}{L_{1}} \mathbf{x}+\left(m \frac{2 \pi}{L_{2}}-n \frac{2 \pi}{L_{1}} \cos \psi\right) \frac{1}{\sin \psi} \mathbf{y} .
$$

The free energy (per unit volume $V$ ) of the vortex lattice can be obtained from (1) by taking $\mathbf{R}_{i}(z)=\left(X_{i}, Y_{i}, z\right)$. One has

$$
\frac{F}{V}=\frac{B^{2}}{8 \pi} \sum_{\mathbf{Q}} \widetilde{V}_{z z}(\mathbf{Q})
$$

where $\widetilde{V}_{z z}(\mathbf{k})$ is given by (17). Next, with the help of the flux quantization condition $L_{1} L_{2} \sin \psi=\Phi_{0} / B$, we can express the free energy in term of one variable only, namely, $\rho=L_{1} / L_{2}$,

$$
\begin{gathered}
L_{1}=\left\{\frac{\Phi_{0}}{B} \frac{\rho}{\left[1-(\rho / 2)^{2}\right]}\right\}^{1 / 2}, \\
L_{2}=\left\{\frac{\Phi_{0}}{B} \frac{1}{\rho\left[1-(\rho / 2)^{2}\right]}\right\}^{1 / 2}, \\
\cos \psi=\frac{\rho}{2} .
\end{gathered}
$$

Finally one minimizes $F / V$ with respect to $\rho$. The numerical minimization of the free energy was done by using the routine GOLDEN. ${ }^{15}$ In Fig. 1 we plotted the value of $\rho$, for which $F / V$ is a minimum, as a function of $\theta$ for $\kappa=60$, $\epsilon=5$, and several values of the induction $b=B / H_{c 2}$ in units of the upper critical field $H_{c 2}$. We find agreement with the results of Ref. 3.

We are now in a position to study the elastic properties of the vortex chain lattice. From (24) we can find all the elastic moduli. For isotropic superconductors there are three independent elastic constants: a compression, a tilt, and a shear. For anisotropic superconductors this number increases dramatically. ${ }^{14,16}$ However, similarly to the isotropic case, the shear modulus is the elastic constant which holds more important information about the vortex lattice. The shear modulus is lattice structure dependent, whereas the other elastic constants can be obtained within the continuum limit in which the lattice is replaced by a liquid of vortices. [This limit is obtained from (24) by considering only the $\mathbf{Q}=0$ contribution to this equation.] So we consider only shear deformations. For anisotropic superconductors there is more than one shear modulus (one "easy" and one "hard") and their definitions are ${ }^{10}$ 


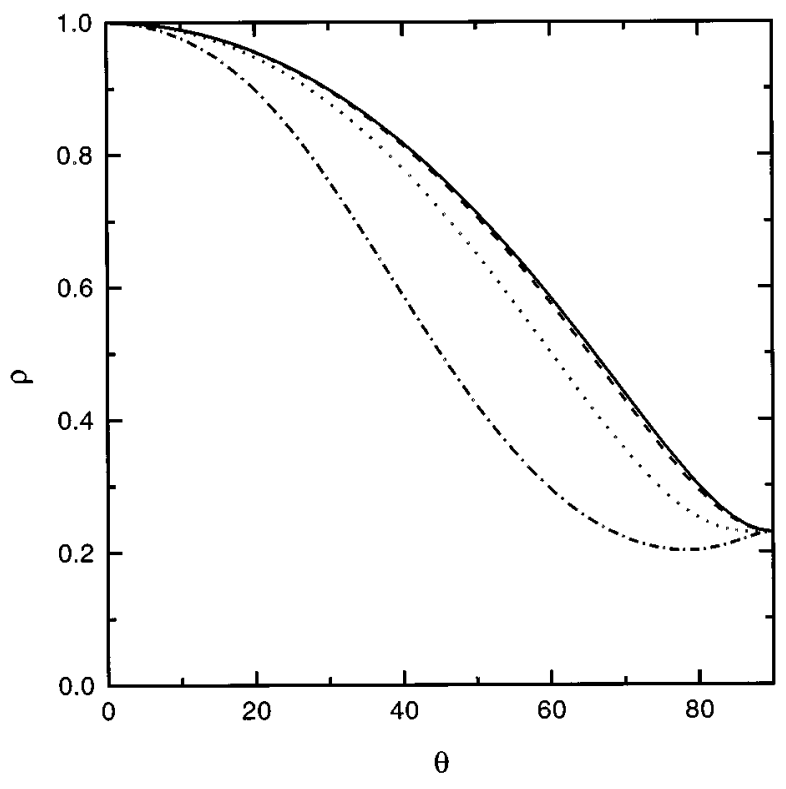

FIG. 1. Plot of $\rho$ against $\theta$ for $\kappa=60, \epsilon=5$, and $b=$ high induction (solid line), and $b=0.005$ (dashed line), $b=0.0005$ (dotted line), and $b=0.0001$ (dot-dashed line).

$$
\begin{aligned}
& c_{66}^{(e)}(\theta)=\lim _{k \rightarrow 0} \frac{\Omega_{-}(0, k, 0)}{k^{2}}, \\
& c_{66}^{(h)}(\theta)=\lim _{k \rightarrow 0} \frac{\Omega_{+}(k, 0,0)}{k^{2}},
\end{aligned}
$$

where $\Omega_{-}(\mathbf{k})$ is the transverse and $\Omega_{+}(\mathbf{k})$ the longitudinal eigenmode (eigenvalue) of $\Phi_{\alpha \beta}(\mathbf{k})$,

$$
\begin{aligned}
\Omega_{ \pm}(\mathbf{k})= & \frac{1}{2}\left[\Phi_{x x}(\mathbf{k})+\Phi_{y y}(\mathbf{k})\right. \\
& \left. \pm \sqrt{\left[\Phi_{x x}(\mathbf{k})-\Phi_{y y}(\mathbf{k})\right]^{2}+4 \Phi_{x y}(\mathbf{k}) \Phi_{y x}(\mathbf{k})}\right] .
\end{aligned}
$$

In Fig. 2, a plot of the easy shear modulus (normalized to its value at $\theta=0$ ) against $\theta$ is shown for $\kappa=50, \epsilon=5$, and several values of $b$. As one can see, by decreasing the induction, the easy shear modulus will no longer have a decreasing monotonic behavior from $\theta=0$ to $\theta=90^{\circ}$. Instead, it will develop a local maximum and local minimum. Similar behavior occurs with the hard shear modulus (normalized to its value at $\theta=0$ ) as can be seen from Fig. 3 where the same parameters were used; for sufficient low induction a minimum appears in the interval $0<\theta<90^{\circ}$.

This change of behavior may lead the easy and hard shear moduli to cross with one another. This in fact happens, as can be seen in Fig. 4 where the values $\kappa=50, \epsilon=5$, and $b=0.0002$ were used. In a subinterval within $0<\theta<90^{\circ}$ the easy shear modulus becomes harder and the hard shear modulus softer.

We also investigated the stability of the vortex chain lattice. As an indication of instability of the lattice we consider the existence of negative eigenvalue. In Fig. 5 we depict $c_{66}^{(h)}(\theta) / c_{66}^{(h)}(0)$ for $\kappa=50, b=0.0003$, and two different values of the anisotropy. For $\epsilon=5$ this quantity remains always positive. Nevertheless, for $\epsilon=60$, the hard shear moduli

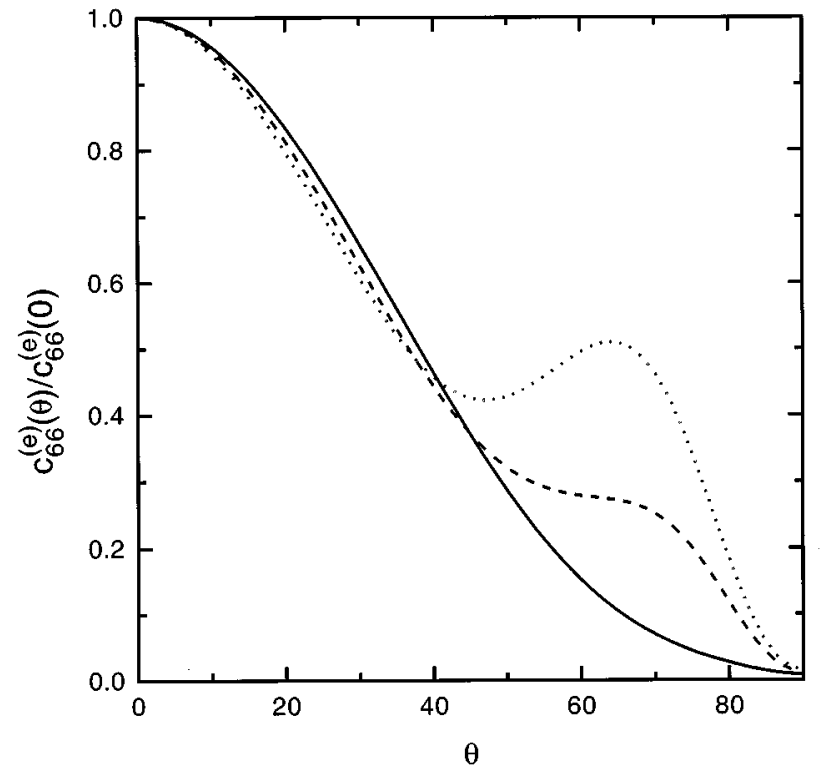

FIG. 2. The easy shear modulus $c_{66}^{(e)}(\theta)$ normalized to its value at $\theta=0$ for $\kappa=50, \epsilon=5$, and $b=0.005$ (solid line), and $b=0.0005$ (dashed line), and $b=0.0003$ (dotted line).

change its sign in the high-angle region. In principle, this would indicate a structural instability of the vortex chain lattice. However, this result should be carefully interpreted since in the high-angle region London theory, as used in the present work, may not be reliable and inhomogeneity of the material has to be accounted for explicitly. ${ }^{17}$

\section{SUMMARY}

In summary we have found two representations for the interaction (tensor) potential for London vortex lines, namely, Eqs. (5)-(9) which should be used in situations where it is more appropriate to work in real space and Eqs.

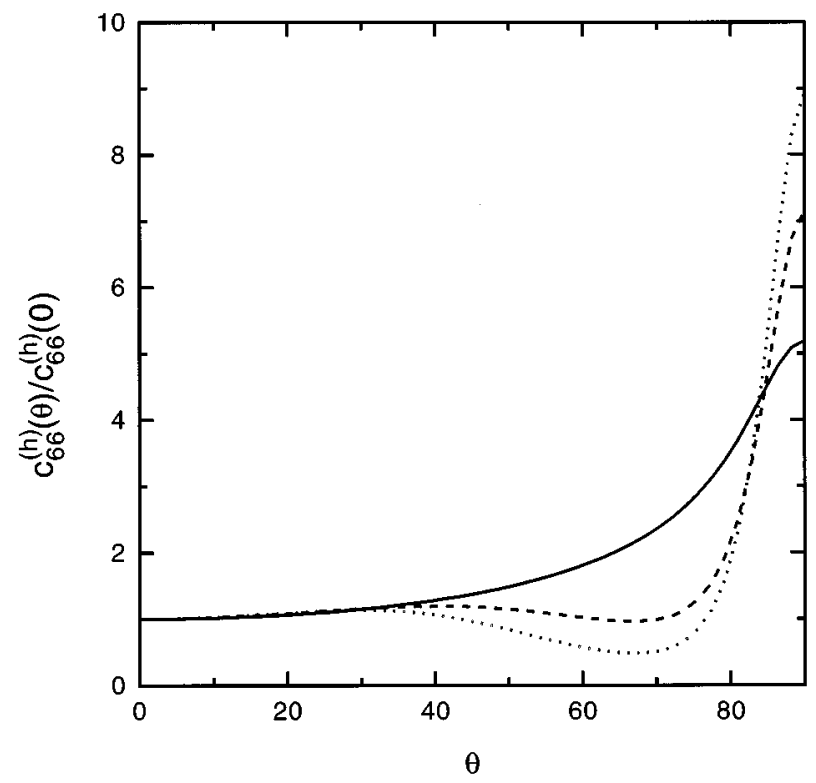

FIG. 3. The hard shear modulus $c_{66}^{(h)}(\theta)$ normalized to its value at $\theta=0$ for $\kappa=50, \epsilon=5$, and $b=0.005$ (solid line), and $b=0.0005$ (dashed line), and $b=0.0003$ (dotted line). 


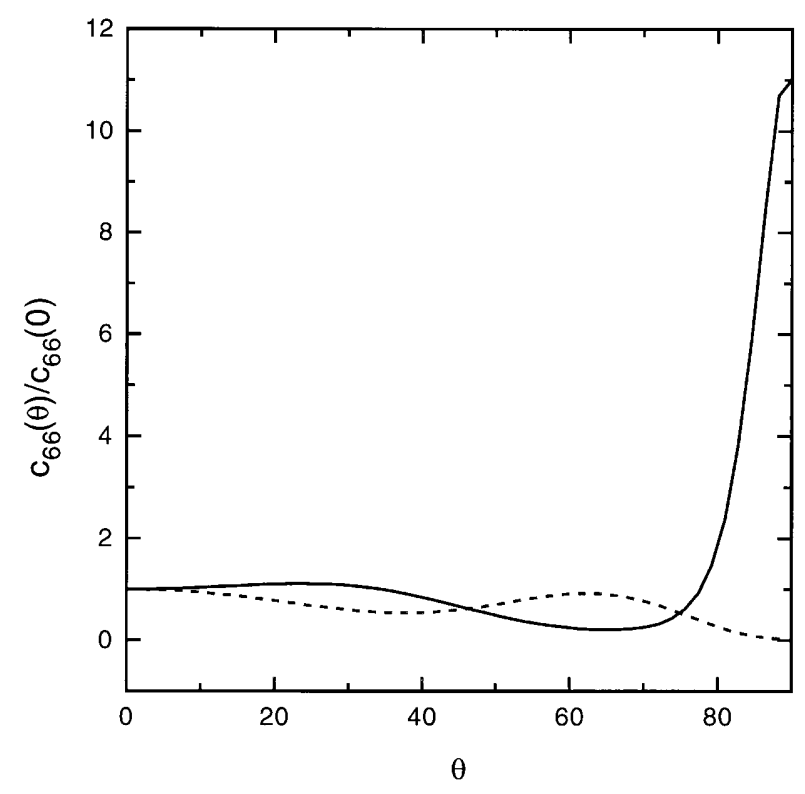

FIG. 4. The hard and easy shear moduli, solid and dashed lines, respectively, for $\kappa=50, \epsilon=5$, and $b=0.0002$. They are normalized to their values at $\theta=0$ which are the same at this angle.

(15)-(20) which should be used in situations where it is more appropriate to work in reciprocal space. This second representation of the potential was used to evaluate the shear moduli of the vortex chain lattice.

\section{ACKNOWLEDGMENTS}

The author thanks CNPq-Conselho Nacional de Desenvolvimento Científico e Tecnológico-Brazil for financial support.

\section{APPENDIX}

We dedicate this appendix to the derivation of (5)-(9) in some detail. The evaluation of the isotropic part of the Lon-

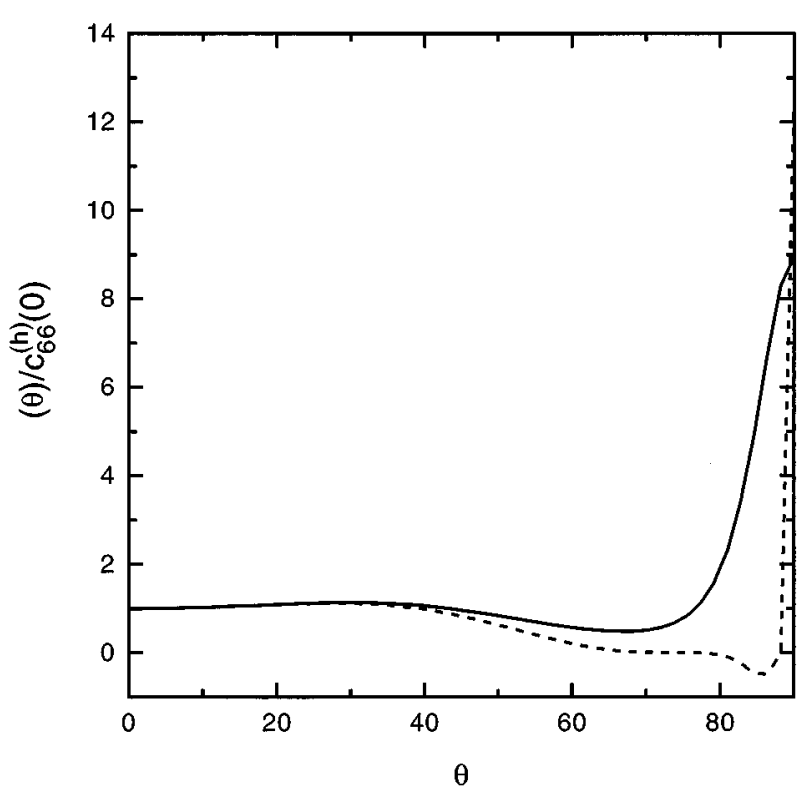

FIG. 5. The hard shear modulus for $\kappa=50, b=0.0003$, and $\epsilon=5$ (solid line) and $\epsilon=60$ (dashed line). don potential is immediate and requires no special technique,

$$
\begin{aligned}
V_{1}^{\alpha \beta}(\mathbf{r}) & =\int \frac{d^{3} k}{(2 \pi)^{3}} \frac{\delta_{\alpha \beta}}{1+\Lambda_{1} k^{2}} e^{i \mathbf{k} \cdot \mathbf{r}} \\
& =\frac{\delta_{\alpha \beta}}{4 \pi \Lambda_{1}} \frac{e^{-r / \sqrt{\Lambda_{1}}}}{r}=\frac{\delta_{\alpha \beta}}{4 \pi \lambda_{a b}^{2}} \frac{e^{-r / \lambda_{a b}}}{r}
\end{aligned}
$$

The anisotropic part of $V_{\alpha \beta}(\mathbf{r})$ can be calculated via the definition of the auxiliary integral (22),

$$
\begin{aligned}
V_{2}^{\alpha \beta}(\mathbf{r}) & =-\Lambda_{2} \int \frac{d^{3} k}{(2 \pi)^{3}} \frac{q_{\alpha} q_{\beta} e^{i \mathbf{k} \cdot \mathbf{r}}}{\left(1+\Lambda_{1} k^{2}\right)\left(1+\Lambda_{1} k^{2}+\Lambda_{2} q^{2}\right)} \\
& =\left\{\left[\nabla[(\nabla I(\mathbf{r})) \times \hat{\mathbf{c}}]_{\alpha}\right] \times \hat{\mathbf{c}}\right\}_{\beta} .
\end{aligned}
$$

Next we make the following change of variables:

$$
\begin{gathered}
k_{x}=\cos \theta k_{x}^{\prime}-\sin \theta k_{z}^{\prime}, \\
k_{y}=k_{y}^{\prime}, \\
k_{z}=\sin \theta k_{x}^{\prime}+\cos \theta k_{z}^{\prime} .
\end{gathered}
$$

For this transformation the volume in reciprocal space, $d^{3} k=d^{3} k^{\prime}$. The idea of this transformation is to bring $\left(1+\Lambda_{1} k^{2}\right)\left(1+\Lambda_{1} k^{2}+\Lambda_{2} q^{2}\right)$ into a form which is cylindrically symmetric. The wave vector $\mathbf{q}=\mathbf{k} \times \hat{\mathbf{c}}$ becomes

$$
\begin{gathered}
q_{x}=\cos \theta k_{y}^{\prime}, \\
q_{y}=k_{x}^{\prime}, \\
q_{z}=\sin \theta k_{y}^{\prime},
\end{gathered}
$$

and $q^{2}=\left(k_{x}^{\prime}\right)^{2}+\left(k_{y}^{\prime}\right)^{2}=\left(k_{\perp}^{\prime}\right)^{2}$.

Now define $\mathbf{r}^{\prime}$ as

$$
\begin{gathered}
x^{\prime}=\cos \theta x+\sin \theta z, \\
y^{\prime}=y, \\
z^{\prime}=-\sin \theta x+\cos \theta z .
\end{gathered}
$$

Hence, dropping the primes on $\mathbf{k}^{\prime}$ the auxiliary integral assumes the form

$$
\begin{aligned}
I(\mathbf{r})= & \Lambda_{2} \int \frac{d^{3} k}{(2 \pi)^{3}} \\
& \times \frac{e^{i \mathbf{k} \cdot \mathbf{r}^{\prime}}}{\left(1+\Lambda_{1} k_{\perp}^{2}+\Lambda_{1} k_{z}^{2}\right)\left[1+\left(\Lambda_{1}+\Lambda_{2}\right) k_{\perp}^{2}+\Lambda_{1} k_{z}^{2}\right]} .
\end{aligned}
$$

After angular integration one has 


$$
\begin{aligned}
I(\mathbf{r})= & \int_{-\infty}^{+\infty} \frac{d k_{z}}{(2 \pi)^{2}} \frac{\cos \left(k_{z} z^{\prime}\right)}{1+\Lambda_{1} k_{z}^{2}} \\
& \times \int_{0}^{\infty} d k_{\perp}\left[\frac{\left(\Lambda_{1}+\Lambda_{2}\right) k_{\perp} J_{0}\left(k_{\perp} \rho^{\prime}\right)}{1+\left(\Lambda_{1}+\Lambda_{2}\right) k_{\perp}^{2}+\Lambda_{1} k_{z}^{2}}\right. \\
& \left.-\frac{\Lambda_{1} k_{\perp} J_{0}\left(k_{\perp} \rho^{\prime}\right)}{1+\Lambda_{1} k_{\perp}^{2}+\Lambda_{1} k_{z}^{2}}\right]
\end{aligned}
$$

where

$$
\begin{aligned}
\left(\rho^{\prime}\right)^{2} & =\left(x^{\prime}\right)^{2}+\left(y^{\prime}\right)^{2} \\
& =(\cos \theta x+\sin \theta z)^{2}+y^{2} \\
& =x^{2}+y^{2}+z^{2}-(\cos \theta z-\sin \theta x)^{2} \\
& =r^{2}-(\mathbf{r} \cdot \hat{\mathbf{c}})^{2}=(\mathbf{r} \times \hat{\mathbf{c}})^{2}
\end{aligned}
$$

Performing a $k_{\perp}$ integration one obtains

$$
\begin{aligned}
I(\mathbf{r}) & =\int_{-\infty}^{+\infty} \frac{d k_{z}}{(2 \pi)^{2}} \frac{\cos \left(k_{z} z^{\prime}\right)}{1+\Lambda_{1} k_{z}^{2}}\left[K_{0}\left(z_{1}\right)-K_{0}\left(z_{2}\right)\right] \\
& =I_{1}(\mathbf{r})-I_{2}(\mathbf{r}),
\end{aligned}
$$

where $z_{i}=\alpha_{i} \sqrt{1+\Lambda_{1} k_{z}^{2}}, \alpha_{i}=a_{i} \rho^{\prime}, a_{1}=1 / \sqrt{\Lambda_{1}+\Lambda_{2}}$, and $a_{2}$ $=1 / \sqrt{\Lambda_{1}}$. Here $K_{0}(x)$ is the modified Bessel function of order zero. To proceed we need to calculate the gradient of the auxiliary integral,

$$
\nabla I_{i}(\mathbf{r})=\nabla \alpha_{i} \frac{\partial I_{i}}{\partial \alpha_{i}}+\nabla z^{\prime} \frac{\partial I_{i}}{\partial z^{\prime}}
$$

Since $z^{\prime}=\mathbf{r} \cdot \hat{\mathbf{c}}, \nabla z^{\prime}=\hat{\mathbf{c}}$. Then

$$
\nabla I_{i}(\mathbf{r}) \times \hat{\mathbf{c}}=\nabla \alpha_{i} \times \hat{\mathbf{c}} \frac{\partial I_{i}}{\partial \alpha_{i}}
$$

and

$$
\begin{aligned}
\frac{\partial I_{i}}{\partial \alpha_{i}}= & -\frac{1}{2 \pi^{2}} \int_{0}^{\infty} d k_{z} \frac{\cos \left(k_{z} z^{\prime}\right)}{\sqrt{1+\Lambda_{1} k_{z}^{2}}} K_{1}\left(\alpha_{i} \sqrt{1+\Lambda_{1} k_{z}^{2}}\right) \\
= & -\frac{1}{2 \pi^{2} \sqrt{\Lambda_{1}}} \sqrt{\frac{\pi}{2} \frac{1}{\alpha_{i}}}\left[\alpha_{i}^{2}\right. \\
& \left.+\left(z^{\prime}\right)^{2} / \Lambda_{1}\right]^{1 / 4} K_{1 / 2}\left[\sqrt{\alpha_{i}^{2}+\left(z^{\prime}\right)^{2} / \Lambda_{1}}\right]
\end{aligned}
$$

where

$$
K_{\overline{2}}^{1}(x)=\sqrt{\frac{\pi}{2 x}} e^{-x} .
$$

Thus, the last equation can be simplified to

$$
\frac{\partial I_{i}}{\partial \alpha_{i}}=-\frac{1}{4 \pi \sqrt{\Lambda_{1}}} \frac{e^{-\sqrt{\alpha_{i}^{2}+\left(z^{\prime}\right)^{2} / \Lambda_{1}}}}{\alpha_{i}},
$$

so that the gradient becomes

$$
\nabla I_{i}(\mathbf{r}) \times \hat{\mathbf{c}}=-\frac{1}{4 \pi \sqrt{\Lambda_{1}}} \frac{\nabla \alpha_{i}}{\alpha_{i}} \times \hat{\mathbf{c}} e^{-\sqrt{\alpha_{i}^{2}+\left(z^{\prime}\right)^{2} / \Lambda_{1}}}
$$

According to the definition of $\alpha_{i}$ above, one can easily show that

$$
\frac{\nabla \alpha_{i}}{\alpha_{i}}=\frac{\nabla \alpha_{i}^{2}}{2 \alpha_{i}^{2}}=\frac{\mathbf{r}-(\mathbf{r} \cdot \hat{\mathbf{c}}) \hat{\mathbf{c}}}{(\mathbf{r} \times \hat{\mathbf{c}})^{2}}
$$

which implies

$$
\nabla I_{i}(\mathbf{r}) \times \hat{\mathbf{c}}=-\frac{1}{4 \pi \sqrt{\Lambda_{1}}} \frac{\mathbf{r} \times \hat{\mathbf{c}}}{(\mathbf{r} \times \hat{\mathbf{c}})^{2}} e^{-\sqrt{\alpha_{i}^{2}+\left(z^{\prime}\right)^{2} / \Lambda_{1}}} .
$$

By inserting this result into (A2) we find $V_{2}^{\alpha \beta}(\mathbf{r})$.

Notice that the results of Sec. II could be derived in a much more direct manner, although not so obvious. The procedure is as follows. Take (5)-(9) and (15)-(20) with $\theta=0$. Let us denote these expressions by $V_{\alpha \beta}^{\mathrm{SB}}(\mathbf{r})$ (Ref. 9) and $\widetilde{V}_{\alpha \beta}^{\mathrm{CDA}}(\mathbf{k})$ (Ref. 12), respectively. Then, (5)-(9) and $(15)-(20)$ are related to these expressions through the equations

$$
\begin{aligned}
& \stackrel{\leftrightarrow}{V}(\mathbf{r})=\stackrel{\leftrightarrow}{R^{T}} \cdot \stackrel{\leftrightarrow}{V} \mathrm{SB}(\widetilde{\mathbf{r}}) \cdot \stackrel{\leftrightarrow}{R}, \quad \widetilde{\mathbf{r}}=\stackrel{\leftrightarrow}{R^{T}} \cdot \mathbf{r} \cdot \stackrel{\leftrightarrow}{R}, \\
& \stackrel{\leftrightarrow}{V}(\mathbf{k})=\stackrel{\leftrightarrow}{R^{T}} \cdot \stackrel{\widetilde{\leftrightarrow}}{\mathrm{CDA}}(\widetilde{\mathbf{k}}) \cdot \stackrel{\leftrightarrow}{R}, \widetilde{\mathbf{k}}=\stackrel{\leftrightarrow}{R^{T}} \cdot \mathbf{k} \cdot \stackrel{\leftrightarrow}{R},
\end{aligned}
$$

where $\stackrel{\leftrightarrow}{R}$ is the rotation matrix. Both the interaction potential and its argument (either $\mathbf{r}$ or $\mathbf{k}$ ) are changed:

$$
\stackrel{\leftrightarrow}{R}=\left(\begin{array}{ccc}
\cos \theta & 0 & \sin \theta \\
0 & 0 & 0 \\
-\sin \theta & 0 & \cos \theta
\end{array}\right)
$$

${ }^{1}$ I. V. Grigorieva, J. W. Steeds, G. Balakrishnan, and D. M. Paul, Phys. Rev. B 51, 3765 (1995).

${ }^{2}$ C. A. Bolle, P. L. Gammel, D. J. Bishop, D. B. Mitzi, and A. Kapitulnik, Phys. Rev. Lett. 66, 2270 (1991).

${ }^{3}$ L. L. Daemen, L. J. Campbell, and V. G. Kogan, Phys. Rev. B 46, 3631 (1993).
${ }^{4}$ L. J. Campbell, M. M. Doria, and V. G. Kogan, Phys. Rev. B 48, 2439 (1988)

${ }^{5}$ V. G. Kogan, N. Nakagawa, and S. L. Thiemann, Phys. Rev. B 42, 2631 (1990).

${ }^{6}$ A. Sudbø and E. H. Brandt, Phys. Rev. Lett. 68, 1758 (1992).

${ }^{7}$ W. Barford and J. M. F. Gunn, Physica C 156, 515 (1988). 
${ }^{8}$ E. H. Brandt, Physica C 165 \& 166, 1129 (1990).

${ }^{9}$ A. Sudb $\varnothing$ and E. H. Brandt, Phys. Rev. B 43, 10482 (1991).

${ }^{10}$ A. Sudb $\varnothing$ and E. H. Brandt, Phys. Lett. 67, 3176 (1991).

${ }^{11}$ E. Z. da Silva and E. Sardella (unpublished).

${ }^{12}$ G. Carneiro, M. M. Doria, and S. C. B. de Andrade, Physica C 203, 167 (1992).

${ }^{13}$ E. Sardella and M. A. Moore, Phys. Rev. B 48, 9664 (1993).

${ }^{14}$ E. Sardella, Phys. Rev. B 45, 3141 (1992).
${ }^{15}$ W. H. Press, B. P. Flannery, S. A. Teukolsky, and W. T. Vetterling, Numerical Recipes (Cambridge University Press, Cambridge, England, 1992).

${ }^{16}$ G. Blatter, M. V. Feigel'man, V. G. Geshkenbein, A. I. Larkin, and V. M. Vinokur, Rev. Mod. Phys. 65, 1125 (1994).

${ }^{17}$ L. N. Bulaevskii, M. Ledvij, and V. G. Kogan, Phys. Rev. B 46, 366 (1992). 